\title{
Câncer de mama gestacional: enfoque diagnóstico e terapêutico
}

\author{
Gestational breast cancer: diagnostic and therapeutic approach \\ Cáncer de mama gestacional: abordaje diagnóstico y terapéutico
}

Lael Andrade Menezes Filho ${ }^{1 *}$, Amanda Gabriele Camargo Bom², Camila Rafaela da Silva Ferreira ${ }^{3}$, Jaqueline Maria de Azevedo Chagas ${ }^{4}$, Letícia Christiane Huida dos Santos ${ }^{5}$, Letícia Farias Oliveira ${ }^{6}$, Marcella Ferreira Pantuzza ${ }^{7}$, Matheus Silva Meireles Ferreira ${ }^{6}$, Rafaela Rodrigues Siqueira ${ }^{8}$, Tatiane Carolina Batista Nacif Roza ${ }^{9}$.

\section{RESUMO}

Objetivo: Descrever a ocorrência do câncer de mama em gestantes, bem como discutir as principais formas de diagnóstico e de tratamento, a partir de uma revisão narrativa de literatura. Revisão bibliográfica: $O$ câncer de mama em gestantes, apesar de raro, é o tipo mais comum de câncer diagnosticado neste período. As alterações fisiológicas e anatômicas que ocorrem durante a gravidez podem fazer com que o diagnóstico ocorra tardiamente e com pior prognóstico. Os procedimentos para o diagnóstico e estadiamento da neoplasia devem levar em consideração a capacidade danosa para o feto, em virtude da radiação dos equipamentos e materiais de contraste, assim, a ultrassonografia é o exame de imagem padrão ouro. Em relação ao tratamento, a mastectomia e a dissecção dos linfonodos axilares fazem parte do plano terapêutico cirúrgico e a quimioterapia pode ser realizada após o primeiro trimestre. Considerações finais: É essencial que ocorra uma avaliação individualizada, considerando a idade gestacional e o estágio da doença, a fim de que seja instituída a terapia adequada a cada paciente, minimizando os impactos à gestante e ao feto.

Palavras-chave: Antineoplásicos, Gestantes, Neoplasias da mama.

\section{ABSTRACT}

Objective: Describe the occurrence of breast cancer in pregnant women, as well as to discuss the main forms of diagnosis and treatment, from a narrative review of the literature. Literature review: Breast cancer in pregnant women, although rare, is the most common type of cancer diagnosed in this period. The physiological and anatomical changes that occur during pregnancy can lead to a late diagnosis and worse prognosis. The procedures for the diagnosis and staging of the neoplasm must take into account the harmful capacity for the fetus, due to the radiation from the equipment and contrast materials, thus, ultrasonography is the gold standard imaging exam. Regarding treatment, mastectomy and axillary lymph node dissection are part of the surgical therapeutic plan and chemotherapy can be performed after the first trimester. Final considerations: It is essential that an individualized assessment takes place, considering the gestational age and stage of the disease, in order to institute the appropriate therapy for each patient, minimizing the impacts on the pregnant woman and the fetus.

Key words: Antineoplastic agents, Pregnant women, Breast neoplasms.

\footnotetext{
1 Universidade Federal da Bahia (UFBA), Vitória da Conquista - BA. *E-mail: lael.filho@ufba.br

2 Universidade Nove de Julho (UNINOVE), São Paulo - SP.

${ }^{3}$ Faculdade de Medicina de Olinda (FMO), Olinda - PE.

${ }^{4}$ Universidade de Rio Verde (UniRV), Aparecida de Goiânia - GO.

${ }^{5}$ Universidade José do Rosário Vellano (Unifenas-BH), Belo Horizonte - MG.

${ }^{6}$ Centro Universitário Uninovafapi (UNINOVAFAPI), Teresina - PI.

7 Pontifícia Universidade Católica de Minas Gerais (PUC-MG), Betim - MG.

8 Universidade de Taubaté (UNITAU), Taubaté - SP.

${ }^{9}$ Centro Universitário de Caratinga UNEC (UNEC), Caratinga - MG.
} 


\section{RESUMEN}

Objetivo: Describir la ocurrencia de cáncer de mama en gestantes, así como discutir las principales formas de diagnóstico y tratamiento, a partir de una revisión narrativa de la literatura. Revisión de la literatura: El cáncer de mama en mujeres embarazadas, aunque raro, es el tipo de cáncer más común diagnosticado en este período. Los cambios fisiológicos y anatómicos que se producen durante el embarazo pueden llevar a un diagnóstico tardío y a un peor pronóstico. Los procedimientos para el diagnóstico y estadificación de la neoplasia deben tener en cuenta la capacidad dañina para el feto, debido a la radiación del equipo y los materiales de contraste, por lo que la ecografía es el examen de imagen estándar de oro. En cuanto al tratamiento, la mastectomía y la disección de los ganglios linfáticos axilares forman parte del plan terapéutico quirúrgico y la quimioterapia se puede realizar después del primer trimestre. Consideraciones finales: Es fundamental que se realice una valoración individualizada, considerando la edad gestacional y el estadio de la enfermedad, con el fin de instituir la terapia adecuada para cada paciente, minimizando los impactos sobre la gestante y el feto.

Palabras clave: Antineoplásicos, Mujeres embarazadas, Neoplasias de la mama.

\section{INTRODUÇÃO}

O câncer durante a gravidez é um evento relativamente raro, com incidência de 1 caso a cada 1000 gestações. Os tipos de câncer mais comumente diagnosticados no período gestacional são câncer de mama, câncer cervical, linfoma, câncer de ovário, leucemia, câncer colorretal e melanoma (POGGIO F, et al., 2020). O Câncer de Mama Associado à Gravidez (CMAG) é definido como aquele diagnosticado durante a gestação ou em um período de um ano após o parto. Entretanto, devido às mudanças fisiológicas no corpo da mulher durante a gravidez, pode ocorrer o diagnóstico tardio desta neoplasia e, consequentemente, gerar pior prognóstico (ALFASI A e BEN-AHARON I, 2019).

Projeções futuras estimam um aumento significativo na taxa de incidência do CMAG, associado ao fato de que as mulheres têm postergado cada vez mais a gestação. Nesse viés, um estudo norte-americano identificou que a idade média das gestações, principalmente do primeiro filho, passou de 24,9 anos no ano 2000 para 26,3 anos em 2014. Além disso, um estudo norueguês indicou que as primigestas com idade superior a 35 anos tiveram risco significativamente maior de CMAG em relação às mulheres que nunca conceberam filhos. Outrossim, um terceiro estudo aponta risco quatro vezes maior de CMAG em mulheres com 40 anos ou mais em comparação com aquelas em idades inferiores a 30 anos (SHACHAR SS, et al., 2017; LABRIOLA B, 2019; YU HH, et al., 2017; CUBILLO A, et al., 2021).

A suspeita clínica do CMAG se dá, geralmente, por uma massa na mama avaliada no exame físico, sendo que a grande maioria destas são benignas. As massas mamárias com alterações de pele associadas à duração superior de duas a quatro semanas aumentam a suspeita de malignidade. Porém, os procedimentos para o diagnóstico e estadiamento da neoplasia devem levar em consideração a capacidade danosa para 0 feto, em virtude da radiação dos equipamentos e materiais de contraste. A ultrassonografia é o exame de primeira linha nestes casos, por apresentar alta sensibilidade e poder ser realizado em todos os trimestres gestacionais. O padrão ouro para o estadiamento do CMAG é a biópsia do linfonodo sentinela, pois pressupõe maior segurança oncológica materna (WANG B, et al., 2019; ALPUIM DC, et al., 2020).

Em relação ao tratamento, a mastectomia e a dissecção dos linfonodos axilares fazem parte do plano terapêutico cirúrgico, sendo as considerações anestésicas as maiores preocupações, em virtude das alterações anatômicas e fisiológicas na gestação. Outra alternativa é a quimioterapia, a qual deve ser realizada de acordo com as diretrizes para as pacientes não grávidas com câncer de mama. Diante disso, esse tipo de intervenção está atrelado ao momento do diagnóstico de câncer de mama e só é indicado após o primeiro trimestre, visto que antes desse período a exposição aos agentes citotóxicos pode afetar o desenvolvimento fetal e aumentar consideravelmente o risco de abortos espontâneos e de malformações congênitas (SHACHAR SS, et al., 2017). 
A terapia endócrina, por sua vez, só é recomendada após o parto e deve ser avaliada em relação ao risco e ao benefício, com o intuito de decidir se haverá o adiamento ou não do tratamento em prol da amamentação. Além disso, a radioterapia tem contraindicação absoluta durante a gestação devido aos efeitos teratogênicos e até letais para o feto e, também, não é recomendada no período da lactação devido ao aumento da toxicidade da pele (POGGIO F, et al., 2020).

Ademais, o CMAG é uma circunstância difícil e desgastantepara as pacientes e seus familiares devido à delicada condução de tratamento e da complexidade na escolha da terapia mais adequada para a mãe e o bebê. Dessa forma, a abordagem da conduta deve ser feita por uma equipe de especialistas multidisciplinar e o tratamento recomendado deve ser discutido com a paciente e sua família, considerando a idade gestacional, as características clínico-patológicas, o prognóstico do câncer, os riscos para o feto e a vontade da mulher (MONTEIRO D, et al., 2013; POGGIO F, et al., 2020).

Diante desse contexto, o presente estudo tem como objetivo descrever a ocorrência do câncer de mama em gestantes, bem como discutir as principais formas de diagnóstico e de tratamento, a partir de uma revisão narrativa de literatura.

\section{REVISÃO BIBLIOGRÁFICA}

O câncer de mama constitui o tipo mais comum de câncer que é diagnosticado durante a gestação, ocorrendo de um a quatro casos por 10.000 gestações. Sabe-se que mudanças que ocorrem durante a gestação como a proliferação progressiva do tecido glandular, alterações na textura e tamanho das mamas, bem como ocorrência de secreção oriunda do mamilo, apesar de fisiológicas, podem dificultar ou retardar a detecção de massas tumorais e, consequentemente, atrasar no diagnóstico. Assim, observa-se que o prognóstico de mulheres com CMAG é precário quando comparado ao câncer de mama de forma geral (ALFASI A e BEN-AHARON I, 2019; GUIMARÃES RF e SPAUTZ CC, 2014).

O CMAG possui relação com mutações não silenciosas e expressão gênica modificada. Dentre as alterações, possui expressão aberrante de genes reguladores de apoptose, supressores de tumor, oncogenes, reguladores de transcrição relacionados ao mecanismo de reparo de DNA, resposta imune, proliferação celular, além de modificações proteicas. Assim, o perfil genômico é composto por padrões mutacionais diversos, o qual explica as características clínicas mais agressivas e de pior prognóstico (KORAKITI AM, et al., 2020).

Casos por hereditariedade representam de 5 a $10 \%$ dos pacientes, no qual os tumores de maior relação com o fator genético são o câncer de mama, ovário, tireoide, olhos e rins (CASWELL-JIN JL, et al., 2017). Por consequência, é observado que um pequeno grupo de mulheres apresenta positividade para receptores de estrogênio, sendo mais comuns em jovens e minimamente encontrados em pacientes com Câncer de mama associado à gravidez. Por outro lado, tumores HER2 positivos são comumente mais frequentes em mulheres grávidas, estando diretamente associado a um pior prognóstico (LEE GE, et al., 2019).

Algumas pesquisas explicitam que a gravidez tardia, preferencialmente gestações após 30 anos, promovem maior exposição ao estímulo estrogênico e está diretamente relacionado com a ocorrência de câncer de mama. Por outro lado, a idade também atua como fator de risco uma vez que aproximadamente $70 \%$ dos tumores são descobertos em mulheres acima de 50 anos de idade. Não obstante, a menarca precoce, menopausa tardia, nuliparidade, exposição a radiação, sobrepeso, obesidade, sedentarismo e histórico familiar de câncer de mama em parente consanguíneo constituem os fatores de risco de grande relevância para o câncer de mama (PERES VC, et al., 2015).

Os diagnósticos de câncer de mama na gestação são iniciados a partir da suspeita de nódulo palpável. Apesar disso, $80 \%$ dos nódulos palpáveis durante a gestação são benignos, entre eles o fibroadenoma, alterações fibrocísticas e galactocele (SHACHAR SS, et al., 2017). A ultrassonografia é o exame de imagem padrão ouro para avaliação de massa mamária, e possui melhor acurácia em mamas de alta densidade. A sensibilidade da USG pode chegar a 100\% em comparação a mamografia, que é em torno de $78 \%-100 \%$. Entretanto, por se tratar de um estudo de imagem examinador-dependente podem gerar resultados falso- 
negativo principalmente em pacientes mais jovens que apresentam mamas de alta densidade (MARTINEZ MT, et al., 2018).

A Ressonância Magnética (RM) é contraindicada na propedêutica de investigação devido aos potenciais efeitos no concepto. O Gadolínio utilizado no procedimento, pode atravessar a placenta e permanecer no líquido amniótico sendo ingerido pelo feto e permanecer na circulação sanguínea fetal. Além disso, o processo de realização do exame pode gerar danos indiretos através da interrupção do fluxo uterino, já que sua realização deve ser realizada em posição de prona (ROJAS KE, et al., 2019). Em situações especiais como metástase óssea e hepática pode ser realizado para o estadiamento RM sem contraste (SHACHAR SS, et al., 2017; WANG B, et al., 2019).

O National Institute for Health and Clinical Excellence (NICE) propõe que a tomografia com emissão de pósitrons (PET-SCAN) não deve ser utilizada em gestantes ou lactantes na investigação ou estadiamento do câncer de mama rotineiramente devido a exposição a radiação e contraste. Na suspeita de sinais ou sintomas para doença metastática, como dor óssea, deve-se considerar cintigrafía para investigar focos de neoplasia. O pulmão é o sítio de metástase mais comum do câncer de mama seguida do fígado. Pacientes com clínica respiratória podem ser avaliados a possibilidade de Tomografia de tórax com baixa carga de contraste. A utilização de RM de crânio deve ser aplicada em todas as pacientes com sinais e sintomas neurológicos e considerar fortemente naquelas com tumores triplo-negativos e HER2 positivo mesmo se assintomáticas (NICE, 2018).

A biópsia com agulha é o padrão ouro para determinar grau histológico, status receptor e epistatus do receptor 2 do fator de crescimento dérmico (HER2). Grande parte das neoplasias diagnosticadas são do tipo carcinomas ductais, com receptor de estrogênio negativo, com alto grau de invasão linfovascular. Os carcinomas medular, mucinoso misto, carcinoma tubular e de células escamosas podem ser observados na gestante em menor proporção (WANG B, et al., 2019).

Se a biopsia confirmar a existência de carcinoma, o estadiamento inicial deve incluir a história clínica detalhada da paciente, enfatizando neoplasias em parentes de primeiro grau, exame físico completo, painel laboratorial completo, incluindo fosfatase alcalina, radiografia de abdômen com blindagem torácica e USG de fígado (MARTíNEZ MT, et al., 2018). O estadiamento envolve o grau de diferenciação histológica, localização, tamanho, disseminação local ou a distância, status do receptor e a expressão da proteína HER2 na membrana (NATIONAL INSTITUTE FOR HEALTH AND CLINICAL EXCELLENCE GUIDELINES, 2018).

As recomendações para o manejo do câncer de mama durante a gravidez são as mesmas que as recomendadas pelas diretrizes às neoplasias mamárias em mulheres jovens não grávidas, e devem considerar as particularidades clínicas da paciente, bem como a idade gestacional, a data prevista para o parto e as suas preferências (POGGIO F, et al., 2020).

A biópsia do linfonodo sentinela deve ser realizada quando indicado e, apesar do risco de linfedema crônico, é um procedimento considerado seguro para essas pacientes. Destaca-se também a importância de notificar os patologistas que irão analisar o linfonodo sentinela sobre o estado de gravidez da paciente, visto que, nesse estado, a drenagem linfática das mamas sofrem alterações fisiológicas que podem influenciar na interpretação da biópsia (ROJAS KE, et al., 2019; MACDONALD HR, 2020; MARTíNEZ MT, et al., 2018; ALFASI A e BEN-AHARON I, 2019; KNABBEN L e MUELLER MD, 2017).

A abordagem cirúrgica ao câncer de mama também recebe essas mesmas recomendações das diretrizes para mulheres não grávidas. Ela é considerada segura durante toda a gestação, porém é mais indicada após o primeiro trimestre da gravidez em decorrência da associação do uso de anestésicos nesse período ao risco aumentado de aborto espontâneo e baixo peso ao nascer e da preocupação em mitigar os possíveis riscos para a organogênese (SHACHAR SS et al, 2017; LEE GE, et al., 2019) .

Apesar da maioria dos anestésicos serem considerados seguros para as gestantes, a segurança da mãe e do feto devem ser priorizadas, sendo importante considerar a passagem dessas substâncias pela placenta, além das alterações anatômicas e fisiológicas inerentes ao período gestacional e suas consequências para o feto. O principal risco devido aos anestésicos é a depressão do sistema cardiovascular ou do sistema nervoso 
central do feto. Além disso, a paciente com mais de 20 semanas de gestação submetida a esse procedimento deve ser posicionada inclinada lateralmente à esquerda, a fim de diminuir a compressão da veia cava inferior pelo útero e proporcionar um retorno venoso eficaz e a perfusão uterina em decúbito dorsal, amenizando possíveis efeitos para o feto e para a mãe, como hipoxemia ou asfixia por hipoperfusão uterina (ROJAS KE, et al., 2019; SHACHAR SS, et al., 2017).

A opção pela cirurgia conservadora de mama deve considerar o tempo de início da radioterapia, visto que, após o parto, essa terapia adjuvante deve ser adiada, e o seu atraso pode implicar em risco aumentado de recorrência local (POGGIO F, et al., 2020; MACDONALD HR, 2020). Nesse contexto, a escolha entre a cirurgia conservadora da mama e a mastectomia deve ser discutida com o cirurgião, e, em geral, quando o câncer é diagnosticado em estágio inicial, as duas opções são consideradas viáveis, assim como para pacientes não grávidas. Entretanto, devido ao típico atraso no diagnóstico desse tipo de malignidade, a mastectomia é a abordagem escolhida nos casos que necessitam de uma abordagem radical. Além disso, é importante reiterar para a paciente no primeiro trimestre que a abordagem conservadora implica em aumento de risco de recorrência local (ALFASI A e BEN-AHARON I, 2019).

Outrossim, o manejo cirúrgico sempre deve ser realizado com indução de sequência rápida (ISR) e monitoramento fetal. O uso de antibióticos possui indicação cirúrgica usual, além disso a profilaxia trombótica deve ser empregada devido à malignidade e a gravidez aumentaram os eventos trombóticos (MACDONALD HR, 2019). O segundo trimestre é o ideal para realizar essa abordagem com quimioterapia adjuvante, enquanto no primeiro e no terceiro trimestre a gravidade da doença, a semana gestacional e a maturidade fetal devem ser avaliadas, analisando os riscos e benefícios da espera pelo segundo trimestre, ou do aguardo ou aceleração do parto, que também pode ser prematuramente induzido pelo estresse cirúrgico, no caso das gestantes no terceiro trimestre, a fim de tratar a neoplasia no pós parto (SHACHAR SS, et al., 2017).

A reconstrução mamária é viável quando o procedimento de escolha é a mastectomia, entretanto, deve ser adiada até que as mudanças fisiológicas mamárias causadas pela gestação se normalizem. Ademais, esse procedimento também deve ser adiado em decorrência do estado de hipercoagulabilidade causado pela gravidez, que pode aumentar o risco de tromboembolismo venoso em cirurgias prolongadas (ROJAS KE, et al., 2019).

Em relação à quimioterapia, sabe-se que a administração de drogas citotóxicas varia de acordo com a idade gestacional, sendo a quimioterapia indicada com o mesmo protocolo de pacientes não gestantes. Entretanto, a farmacocinética desses fármacos durante a gravidez pode ser modificada por vários fatores como a redução da albumina plasmática, aumento no compartimento de água, e mudanças na oxidação hepática, depuração renal e na motilidade gastrointestinal (BAE SY, et al., 2018).

Durante o primeiro trimestre, a exposição aos quimioterápicos aumenta as chances de anomalias congênitas ou perda fetal. Entretanto, a exposição de determinadas drogas durante o segundo e terceiro trimestres não demonstraram aumentar as taxas de danos fetais. Porém, efeitos na supressão da hematopoiese e restrição do crescimento fetal podem ocorrer e devem ser monitorados durante todo o período de tratamento (MACDONALD HR, 2020; LOIBL S, et al., 2015).

Atualmente, as antraciclinas, ciclofosfamida e regimes baseados em taxanos representam o padrão de cuidados para o tratamento neoadjuvante de pacientes com câncer de mama e os efeitos colaterais mais comuns incluem a neutropenia, úlceras orais, anafilaxia, constipação, taquicardia e celulite (PALUCHSHIMON S, et al., 2020; LABRIOLA B, 2019). No caso das gestantes, a administração de quimioterapia deve ser evitada após as 35 semanas de gestação, pois pode associar-se a mielossupressão, seguida por eventos adversos no momento do parto como sangramento, sepse ou morte (BERVEILLER P, et al., 2019).

O uso do tamoxifeno não é recomendado uma vez que, administrado durante a gravidez ou no período de lactação, pode causar defeitos de malformações craniofaciais, genitália ambígua e morte fetal (CORDEIRO CN e GEMIGNANI ML, 2017). O trastuzumabe também não é indicado durante a gestação pois pode causar riscos de oligodramnia, adramnia, hipoplasia pulmonar fetal e perfusão renal diminuída (GOLLER SS, et al., 2019). 
A radioterapia, por sua vez, deve ser adiada para o pedido pós-parto, já que a exposição à radiação poder causar alterações fetais em todas as fases da gestação e resultar em aborto, malformação e restrição do crescimento fetal (AMANT F, et al., 2019). Se a radioterapia for indicada durante a gravidez, a proteção fetal da pelve deve ser considerada e a blindagem fetal pode diminuir a dose de radiação em $50 \%$ a $75 \%$ (BOTHA et al., 2018; TOESCA A, et al., 2014). Em relação à amamentação, é bem documentado que medicamentos quimioterápicos, hormônios e terapias alvo podem chegar ao leite materno e, dessa maneira, serem passados para o bebê. Desse modo, não é recomendada a amamentação durante a quimioterapia, hormonioterapia ou terapia alvo (LUNDQVIST EA, et al., 2015).

Assim, a escolha das terapias durante o período gestacional é um desafio ético e emocional de grande carga a equipe médica e as pacientes. A avaliação sistematizada e individual, levando em consideração 0 momento da gestação, o estágio da doença e o subtipo histológico são cruciais para elevar os benefícios e minimizar os danos à gestante e ao feto (SHACHAR SS, et al., 2017).

\section{CONSIDERAÇÕES FINAIS}

A partir da análise dos achados da literatura, verificou-se uma baixa incidência de câncer de mama durante a gravidez. Entretanto, torna-se necessária investigar tal neoplasia em caso ocorrência de nódulos ou anormalidades na mama, uma vez que o diagnóstico tardio acarreta em um pior prognóstico. Além disso, diversos métodos diagnósticos que envolvam radiação e materiais de contraste são contraindicados, deixando a ultrassonografia como exame preferencial nestes casos. Ademais, é fundamental avaliar as particularidades clínicas de cada paciente, idade gestacional, estágio da doença e identificar quais são as terapias antineoplásicas indicadas para o manejo do câncer de mama na gestante em cada caso.

\section{REFERÊNCIAS}

1. ALFASI A, BEN-AHARON I. Breast cancer during pregnancy - current paradigms, paths to explore. Molecular Diversity Preservation International, 2019; (11): 1669-1685.

2. ALPUIM DC, et al. Cancer During Pregnancy: How to Handle the Bioethical Dilemmas?-A Scoping Review With Paradigmatic Cases-Based Analysis. Frontiers in oncology; 2020, 10: 598508.

3. AMANT F, et al. Gynecologic cancers in pregnancy: guidelines based on a trird international consensus meeting. Annals of Oncology; 2019; (30): 1601-161.

4. BAE SY, et al. Clinical characteristics and prognosis of pregnancy- associated breast cancer: Poor survival of luminal b subtype. Oncology, 2018; (95): 163-169

5. BERVEILLER P, et al. Hermotherapy in pregnancy: Exploratory study of the effects of paclitaxel on the expression of placental drug transporters. Investigational new drugs, 2019; 37(5): 1075-1085.

6. BOTHA MH, et al.Câncer treatment in Pregnant Women. Contemporary Oncology; 2015; (19): 354- 360.

7. CASWELL-JIN JL, et al. Racial/Ethnic Differences in Multiple-Gene Sequencing Results for Hereditary Cancer Risk. Genetics in Medicine, 2018; 20(2): 234-239.

8. CORDEIRO CN, GEMIGNANI ML. Breast can- cer in pregnancy: Avoiding fetal harm when maternal treatment is necessary. Breast Journal; 2017; 23(2): 200-205.

9. CUBILLO A, et al. Multidisciplinary consensus on cancer management during pregnancy. Clinical \& translational oncology: official publication of the Federation of Spanish Oncology Societies and of the National Cancer Institute of Mexico; 2021, 23(6): 1054-1066.

10. GOLLER SS, et al. Trastuzumab in the Treatment of Pregnant. Geburtsh Frauenheilk; 2019; 79: 618-625.

11. GUIMARÃES RF, SPAUTZ CC. Câncer de mama associado à gestação. Femina, 2014; 42(4): 204-208.

12. KNABBEN L; MUELLER MD. Breast cancer and pregnancy. Hormone Molecular Biology and Clinical Investigation, 2017; 32(1): 1-7.

13. KORAKITI AM, et al. The Genomic Profile of Pregnancy-Associated Breast Cancer: A Systematic Review. Frontiers in Oncology, 2020; 10: 1773.

14. LABRIOLA B. Treatment of Pregnancy-Associated Breast Cancer. Journal of the Advanced Practitioner in Oncology; 2019 10(7): 692-700.

15. LEE, GE, et al. Contemporary Management of Breast Cancer during Pregnancy and Subsequent Lactation in a Multicenter Cohort of Young Women with Breast Cancer. The Breast Journal, 2019; 25(6): 1104-1110.

16. LOIBL S, et al. Breast cancer diagnosed during pregnancy: Adapting recent advances in breast cancer care for pregnant patients. JAMA Oncology, 2015; (1): 1145-1153.

17. LUNDQVIST EA, et al. Princípios da quimioterapia - FIGO Cancer Report. 2015. Disponível em: https://obgyn.onlinelibrary.wiley.com/pb-assets/hub-assets/obgyn/1879-3479_IJGO/translated_content/ijgos146sup-0001-Portuguese-1509630368553.pdf. Acesso em: 23 de julho de 2021. 
18. MACDONALD HR. Pregnancy associated breast cancer. The Breast Journal, 2020; 26(1): 81-85.

19. MARTÍNEZ MT, et al. Breast cancer in pregnant patients: a review of the literature. European Journal of Obstetrics and Gynecology and Reproductive Biology, 2018; 230(1): 222-227.

20. MONTEIRO D, et al. Câncer de mama na gravidez e quimioterapia: revisão sistemática. Revista da Associação Médica Brasileira, 2013; 59(2): 174-180.

21. NATIONAL INSTITUTE FOR HEALTH AND CLINICAL EXCELLENCE GUIDELINES. Early and locally advanced breast cancer: diagnosis and management - NICE. 2018. Disponível em: https://www.nice.org.uk/guidance/ng101. Acesso em: 14 de julho de 2021.

22. PALUCH-SHIMON S, et al. ESO-ESMO 4th International Consensus Guidelines for Breast Cancer in Young Women (BCY4). Annals of oncology: official journal of the European Society for Medical Oncology, 2020; 31(6): 674-696.

23. PERES VC, et al. Breast cancer in women: recurrence and survival at five years. Texto \& Contexto - Enfermagem, 2015; 24(3): 740-747

24. POGGIO F, et al. Update on the Management of Breast Cancer during Pregnancy. Cancers, 2020; $12(12): 3616-3633$.

25. ROJAS KE, et al. A Review of Pregnancy-Associated Breast Cancer: Diagnosis, Local and Systemic Treatment, and Prognosis. Journal of Women's Health, 2019; 28(6): 778-784.

26. SHACHAR SS, et al. Multidisciplinary Management of Breast Cancer During Pregnancy. The Oncologist, 2017; 22(3): 324-334.

27. TOESCA A, et al. Locoregional Treatment of Breast Can- Cer during Pregnancy. Gynecological Surgery. 2014; 11(4): 279-284.

28. WANG B, et al. Clinicopathology characteristics, diagnosis, and prognosis of pregnancy-associated breast cancer. Thoracic Cancer, 2019; (10): 1060-1068.

29. $\mathrm{YU} \mathrm{HH}$, et al. Current management of pregnancy-associated breast cancer. Hong Kong Journal of Emergency Medicine, 2017, 23(4): 387-394. 